\title{
ON SPHERICAL CYCLES ${ }^{1}$
}

\section{SAMUEL EILENBERG}

Given a metric separable space $\Upsilon$, we consider the homology group $B^{n}(\Upsilon)$ obtained using $n$-dimensional singular cycles in $\Upsilon$ with integer coefficients. Every continuous mapping $f \in \Upsilon^{s^{n}}$ of the oriented $n$-dimensional sphere $S^{n}$ into $Y$ defines uniquely an element $h(f)$ of $B^{n}(\Upsilon)$. Clearly if $f_{0}, f_{1} \in \Upsilon^{s^{n}}$ are two homotopic mappings, then $h\left(f_{0}\right)=h\left(f_{1}\right)$.

The homology classes $h(f)$ will be called spherical homology classes. A cycle will be called spherical if its homology class is spherical. ${ }^{2}$

THEOREM 1. If $\Upsilon$ is arcwise connected, the spherical homology classes form a subgroup of $B^{n}(\Upsilon)$.

Let $p \in S^{n}, q \in \Upsilon$, and let $S^{n}=S_{+}^{n}+S_{-}^{n}$ be a decomposition of $S^{n}$ into two hemispheres such that $p \in S_{+}^{n} \cdot S_{-}^{n}$. Consider $f_{0}, f_{1} \in \Upsilon^{n} s^{n}$. It is well known that, replacing if necessary $f_{0}$ and $f_{1}$ by homotopic mappings, we may assume that $f_{0}\left(S_{+}^{n}\right)=q$ and that $f_{1}\left(S_{-}^{n}\right)=q$. Defining $f=f_{0}$ on $S_{-}^{n}$ and $f=f_{1}$ on $S_{+}^{n}$ we clearly have

$$
f \in \Upsilon^{s^{n}}, \quad h(f)=h\left(f_{0}\right)+h\left(f_{1}\right) .
$$

The homology class $h\left(f_{0}\right)+h\left(f_{1}\right)$ is therefore spherical.

Let $M^{r}$ be an $r$-dimensional (finite or infinite) manifold ${ }^{3}$ and $P^{r-n-1}$ $(n>0)$ an at most $(r-n-1)$-dimensional subpolyhedron of $M^{r}$.

THEOREM 2. Every n-dimensional cycle $\gamma^{n}$ in $M^{r}-P^{r-n-1}$ such that $\gamma^{n} \sim 0$ in $M^{r}$ is spherical (with respect to $M^{r}-P^{r-n-1}$ ).

Let $a^{r-n-1}$ be an $(r-n-1)$-dimensional simplex of $M^{r}$ and $b^{n+1}$ the $(n+1)$-cell dual to it. The boundary $\partial b^{n+1}$ is contained in $M^{r}-P^{r-n-1}$ and is a spherical cycle. Since $M^{r}-P^{r-n-1}$ is connected, the spherical homology classes of $B^{n}\left(M^{r}-P^{r-n-1}\right)$ form a group. It follows that each cycle of the form

$$
\partial\left(\sum_{i} \alpha_{i} b_{i}^{n+1}\right)
$$

is a spherical cycle with respect to $M^{r}-P^{r-n-1}$. The cycle $\gamma^{n}$ is homologous in $M^{r}-P^{r-n-1}$ to a cycle of the form $\left(^{*}\right)$. Therefore $\gamma^{n}$ is spherical.

${ }^{1}$ Presented to the Society, April 13, 1940.

${ }^{2}$ Spherical cycles were considered by W. Hurewicz, Proceedings, Akademie van Wetenschappen te Amsterdam, vol. 38 (1935), pp. 521-528.

${ }^{3}$ See K. Reidemeister, Topologie der Polyeder, Leipzig, 1938, p. 151. 
Theorem 3. Let $\gamma^{n}$ be a spherical cycle in $M^{r}$ and let $r>2 n$. Then there is a simplicial homeomorphism ${ }^{4} g \in M^{r^{S^{n}}}$ such that $\gamma^{n} \in h(g)$.

This is an immediate consequence of Theorem 5 below. Using Theorem 2 we obtain the following:

Theorem 4. Given an $n$-cycle ${ }^{n} \subset M^{r}-P^{r-n-1}(r>2 n)$ such that ${ }^{n} \sim 0$ in $M^{r}$, there is a cycle $\gamma_{1}^{n} \subset M^{r}-P^{r-n-1}$ which is a simplicial and homeomorphic image of $S^{n}$ such that $\gamma^{n} \sim \gamma_{1}^{n}$ in $M^{r}-P^{r-n-1}$.

THEOREM 5. Let $Q^{n}$ be a finite $n$-dimensional polyhedron and let $r>2 n$. Every continuous mapping $f \in M^{r^{Q^{n}}}$ can be approached by simplicial homeomorphisms $g \in M^{r^{Q^{n}}}$.

We may admit that the mapping $f$ is simplicial. Let $a_{1}, a_{2}, \cdots, a_{k}$ be the vertices of the complex $f\left(Q^{n}\right)$ and let $\sigma_{1}, \sigma_{2}, \cdots, \sigma_{k}$ be the corresponding stars. ${ }^{5}$ Let us choose $\delta>0$ so that $x \in f\left(Q^{n}\right)$ will imply $\rho\left(x, M^{r}-\sigma_{i}\right)>\delta$ for some $i=1,2, \cdots, k$.

Let $\delta>2 \epsilon>0$. We are going to define a sequence $f=f_{0}, f_{1}, \cdots, f_{k}$ of simplicial maps of $Q^{n}$ into $M^{r}$ such that

(3) $\quad x_{1} \neq x_{2}$ and $f_{i}\left(x_{1}\right)=f_{i}\left(x_{2}\right)=y$ imply $\rho\left(y, M^{r}-\sigma_{i}\right)<\delta \frac{2 k-i}{2 k}$.

Suppose that $f_{0}, f_{1}, \cdots, f_{i-1}$ are already defined. Let

$$
f_{i}(x)=f_{i-1}(x) \text { if } f_{i-1}(x) \in M^{n}-\sigma_{i},
$$

and let $Q_{i}^{k}=f_{i-1}^{-1}\left(\sigma_{i}\right)$.

$M^{r}$ being a manifold, $\sigma_{i}$ is simplicially homeomorphic with a convex $r$-cell in a euclidean $r$-dimensional space. Since $r>2 n$, then using the very well known ${ }^{6}$ procedure of making vertices linearly independent we find a simplicial map $f_{i}\left(Q_{i}^{n}\right) \subset \sigma_{i}$ such that $f_{i}(x)=f_{i-1}(x)$ if $f_{i-1}(x)$ is on the boundary of $\sigma_{i}$ and satisfying (1)-(3).

Taking $g=f_{k}$ it follows from (1) that

$$
|g(x)-f(x)|<\epsilon .
$$

\footnotetext{
${ }^{4}$ With respect to certain simplicial subdivisions of $M^{r}$ and $S^{n}$.

${ }^{5} \sigma_{i}$ consists of all closed simplices of $M^{r}$ containing $a_{i}$.

${ }^{6}$ See for instance W. Hurewicz, Sitzungsberichte der Preussischen Akademie der Wissenschaften, vol. 24 (1933), p. 758.
} 
Now if $x_{1} \neq x_{2}$ and $g\left(x_{1}\right)=g\left(x_{2}\right)$, then according to (2) we have

$$
f_{i}\left(x_{1}\right)=f_{i}\left(x_{2}\right)=y_{i} \quad \text { for } i=0,1, \cdots, k .
$$

Owing to the definition of $\delta$ there is an index $j=0,1, \cdots, k$ such that

$$
\rho\left(y_{0}, M^{n}-\sigma_{j}\right)>\delta .
$$

Combining this with (1) we see that

$$
\rho\left(y_{i}, M^{n}-\sigma_{j}\right)>\delta-\frac{\epsilon i}{k}>\delta-\frac{\delta i}{2 k}=\delta \frac{2 k-i}{2 k} .
$$

Taking $i=j$ we obtain a contradiction with (3).

UNIVERSITY OF MichigAN 